\title{
Indoor Localization Using Multi-Frequency RSS
}

Martin Skoglund, Gustaf Hendeby, Jonas Nygårds, Jouni Rantakokko and Gunnar Eriksson

\author{
Linköping University Post Print
}

\section{Tweet}

N.B.: When citing this work, cite the original article.

(C2016 IEEE. Personal use of this material is permitted. However, permission to reprint/republish this material for advertising or promotional purposes or for creating new collective works for resale or redistribution to servers or lists, or to reuse any copyrighted component of this work in other works must be obtained from the IEEE.

Martin Skoglund, Gustaf Hendeby, Jonas Nygårds, Jouni Rantakokko and Gunnar Eriksson, Indoor Localization Using Multi-Frequency RSS, 2016, Proceedings of the IEEE/ION Position Location and Navigation Symposium.

Postprint available at: Linköping University Electronic Press

http://urn.kb.se/resolve?urn=urn:nbn:se:liu:diva-127138 


\title{
Indoor Localization Using Multi-Frequency RSS
}

\author{
Martin A. Skoglund*, Gustaf Hendeby* Jonas Nygårds ${ }^{\dagger}$ Jouni Rantakokko ${ }^{\ddagger \S}$ Gunnar Eriksson ${ }^{\ddagger}$ \\ * Dept. of Electrical Engineering, Linköping University, Linköping, Sweden \\ $\dagger$ Dept. of Sensor \& EW Systems, Swedish Defence Research Agency (FOI), Linköping, Sweden \\ $\ddagger$ Dept. of Robust Telecommunications, Swedish Defence Research Agency (FOI), Linköping, Sweden \\ $\S$ Dept. of Signal Processing, KTH Royal Institute of Technology, Stockholm, Sweden
}

\begin{abstract}
This paper investigates the usefulness of multifrequency received signal strength (RSS) for indoor localization. A collected set of data from four sites containing 7 frequencies from dual receivers and a high accuracy reference positioning system is presented. The collected data is also made publicly available through ResearchGate. The data is analyzed with respect to spatial variations using Gaussian processes (GP). The results show that there are more rapid signal variations across corridors than along them. The uniqueness of RSS fingerprints is analyzed suggesting that sequences of measurements in smoothing, or smoothing-like, algorithms that can handle temporary position ambiguities are likely the best choice for localization applications.
\end{abstract}

\section{INTRODUCTION}

A reliable and accurate positioning system is expected to significantly improve the safety for first responders and to enhance their operational efficiency [1]. Commercial indoor localization methods often utilize pre-installed infrastructure e.g., WiFi access points or Bluetooth beacons and does also require recording received signal strength (RSS) maps beforehand, i.e., finger printing. Relying on WiFi RSS fingerprints may fail in many first responder scenarios since, e.g., the location is rarely known in advance so that it can be mapped, the electricity in the building may be lost, the building may be subjected to structural damage, or WiFi may not be installed.

Existing dead-reckoning systems still provide insufficient accuracy, especially when considering realistic first responder operations. Foot-mounted inertial navigation systems (INS) are an interesting option for these applications but they may on occasion suffer a heading drift that have a detrimental effect on the accuracy [2]. Hence, in indoor safety-critical applications supporting sensors are needed. Strong signals-of-opportunity (SoOp) are available indoors such as those originating from broadcast FM radio and TV transmitters. Low-cost, small and lightweight receivers can be utilized in order to capture RSS values from these transmitters.

Several simultaneous localization and mapping (SLAM) systems relying on RSS measurements, e.g., WiFi-SLAM [3] and WiFi GraphSLAM [4], estimates the RSS fingerprints onthe-fly or unknown access point locations [5]. A less explored alternative is to use multi-frequency RSS measurements of other opportunistic radio sources, e.g., FM radio [6] and TV stations without pre-surveyed fingerprints.

In [7], FM radio RSS values were recorded at up to 32 frequencies and room-level accuracy was demonstrated by comparing the current RSS vector with stored training RSS vectors and adopting the nearest neighbors (in the signal space) position as the current position. Also, the accuracy could be improved by adding WiFi RSS data, as as well as by including low-level information such as SNR, multipath, and frequency offset estimates if they are available in the receiver.

FM radio localization for room and floor-level localization based on signal fingerprinting has also been examined in [8]. Device uncertainty, i.e., where different units produces varying RSS values, has shown to reduce the accuracy. However, in first responder applications device calibration may be feasible to perform. Temporal variations of the RSS values over the course of several days can reduce the localization accuracy, and it is impractical to recalibrate regularly. Although the FM radio signals are less affected by temporal variation compared to WiFi signals, an approach where the user collects and stores RSS values during the course of the operation is a means to reduce the effects of temporal variations. In [9], a smartphone-based system was developed that encompassed a pedestrian dead-reckoning (PDR) sub-system combined with the opportunistic use of RSS measurements from transmitters at unknown locations in a particle filter approach. The indoor localization system in [10] uses a floor attenuation factor propagation model in favor of Rayleigh fading [11] or Rician distribution [12]. In more recent work [3, 9, 13, 14] Gaussian processes are used to model the RSS, and [15] also models the signals source location.

In our previous work [14] a RSS SLAM system with with foot mounted (INS) was designed, and shown to be successful. However, the added value of using multi-frequency RSS from opportunistic signals in this SLAM solution is difficult to separate from the contribution of the foot-mounted INS subsystem and the filtering solution itself. Our intention here is therefore rather to take a step back and investigate what potential lies in the RSS measurements from FM radio and TV transmitters alone. This knowledge is quite useful for designing localization solutions since it is then easier to specify the requirements on the other parts of the system, e.g., tolerated drift in an inertial navigation system (INS) or simply for tuning of filter parameters.

In this work we will present datasets and analysis using Gaussian Processes as model with a bearing on the usefulness of RSS for collaborative SLAM.

The paper is organized in the following way. In Sec. II the RSS measurements and the Gaussian processes (GPs) used to model them are described. Sec. III details the experimental 
setup and the collected data are presented in Sec. IV. An analysis of the data and the suitability of the GP to model them is provided in Sec. V. Conclusions are drawn in Sec. VI.

\section{RSS MEASUREMENTS}

There are several reasons to why it is interesting to study the opportunistic use of multi-frequency RSS for indoor localization. FM radio and TV signals are present almost everywhere and may therefore be utilized in e.g., first responder scenarios where pre-installed infrastructure cannot be trusted. Signal fading characteristics depends on frequency, and the surrounding environment, resulting in different RSS maps. These maps combined are naturally more informative than a single map alone [7].

\section{A. Indoor Radio Signals}

A radio signal interacts with the physical environment in an extremely complex manner. It experiences a distance dependent attenuation (path loss) and the radio signal is reflected off different objects, diffracted around obstacles, and scattered off objects. Hence, the receiver will receive many (distorted and delayed) signal components. The constructive and destructive addition of these multipath components is the cause for the rapid fluctuations as a function of spatial displacement of the RSS values that are typical for all non-lineof-sight (NLOS) wireless radio channels which is the typical situation here. This phenomena is called multipath fading. Apart from the multipath fading, also antenna orientation and shadowing affects the measurements. Previous measurements show that effects such as antenna orientation may change the RSS values by $3 \mathrm{dBm}$ at FM frequencies while shadowing by a human close to the antenna may affect the RSS-values by up to $7 \mathrm{dBm}[16]$.

\section{B. Gaussian Processes}

We will here only give a brief description of GPs and its use with RSS for localization purposes. For more detailed expositions, see e.g., [13], or [17] which treats GPs in general. An RSS fingerprint of $n$ frequencies is denoted

$$
\Lambda=\left[\begin{array}{llll}
\Lambda^{1} & \Lambda^{2} & \ldots & \Lambda^{n}
\end{array}\right]^{T},
$$

where each $\Lambda_{i}$ is the RSS for the $i^{\text {th }}$ frequency. A measurement at time instant $k$ is an RSS fingerprint vector, $y_{k}=\left(\begin{array}{lll}y_{k}^{1} & \ldots & y_{k}^{n}\end{array}\right)^{T}$. Each component

$$
y_{k}^{i}=\Lambda_{k}^{i}+e_{k}^{i},
$$

contains an RSS value for a frequency and $e_{k}^{i} \sim \mathcal{N}\left(0, \sigma_{e}^{2}\right)$ is the measurement noise for component $i$. In turn, measurements can be viewed as being generated by a GP

$$
y_{k}^{i}=f_{k}^{i}(x)+e_{k}^{i},
$$

where $f()$ is some function parameterized by $x$. In order to estimate a GP for each frequency a set of training samples consisting of (inputs) positions and (targets) RSS measurements $D=\left(x_{1}, y_{1}\right),\left(x_{2}, y_{2}\right), \ldots,\left(x_{N}, y_{N}\right)=(X, Y)$ without specifying any specific frequency. The idea with GPs is that the targets from different inputs, say $f(x)$ and $f\left(x^{\prime}\right)$, are correlated and that this can be represented with a covariance function (kernel), $\mathcal{K}\left(x, x^{\prime}\right)$, and a mean value function $\mathcal{M}$. The covariance for any two measurement are

$$
\operatorname{cov}\left(y, y^{\prime}\right)=\mathcal{K}\left(x, x^{\prime}\right)+\sigma_{e}^{2} \delta_{x=x^{\prime}},
$$

where $\delta_{x=x^{\prime}}$ is the Dirac-delta function that is 1 if $x=x^{\prime}$ and otherwise 0 . The joint distribution of observed measurements $Y$ and an unobserved (test) RSS value $f\left(x_{*}\right)$ is

$$
\left(\begin{array}{c}
Y \\
f\left(x_{*}\right)
\end{array}\right)=\mathcal{N}\left(\left(\begin{array}{c}
\mu \\
\mu_{*}
\end{array}\right),\left(\begin{array}{cc}
\Sigma & \Sigma_{*} \\
\Sigma_{*}^{T} & \Sigma_{* *}
\end{array}\right)\right),
$$

where $\mu_{i}=\mathcal{M}\left(x_{i}\right)$, for $i=1, \ldots, n$, is the $i^{\text {th }}$ component of $\mu$ and analogously for $\mu_{*}$. The $\Sigma$ matrices denote the covariances between all combinations of test and training inputs. The predictive distribution of the unknown function values given the training samples becomes

$$
\begin{aligned}
f\left(x_{*}\right) & \mid x_{*}, X, Y \\
& \sim \mathcal{N}\left(\mu_{*}+\Sigma_{*}^{T} \Sigma^{-1}(Y-\mu), \Sigma_{* *}-\Sigma_{*}^{T} \Sigma^{-1} \Sigma_{*}\right),
\end{aligned}
$$

in which the computational bottleneck is the computation and inversion the covariance matrix $\Sigma$. This issue will not be addressed here but is of course important, especially in online applications, see e.g., [18, 19].

There is a great deal of flexibility using GPs due to the many options of kernels, mean functions, and hyper-parameters, that still result in a Gaussian posterior distributions. Luckily, the most suitable choice of hyper-parameters values can be done by e.g., maximizing the marginal likelihood with respect to the hyper-parameters [17] as is done in Sec. V.

\section{EXPERIMENT SETUP}

The section describes the hardware that was used in the experiments and the calibration procedure.

\section{A. Radio Receiver}

The receivers cover the frequency ranges $150 \mathrm{kHz}$ to $240 \mathrm{MHz}$ and $420 \mathrm{MHz}$ to $1.9 \mathrm{GHz}$. The receivers use a sampling frequency of $192 \mathrm{kHz}$ and each single-frequency RSS value was recorded over a period of $4 \mathrm{~ms}$. Two receivers and antennas were placed approximately $2 \mathrm{dm}$ apart on a rollerboard and they measured RSS-values in parallel. A prism was placed on the roller-board, between the two antennas, and a laser total station (a Topcon QS3A) provided reference positions during the tests. The laser automatically tracks the prism and estimates the direction and distance to it with high accuracy. The positions of the two antennas were estimated based on the fix lever-arm distance. The reference positions for the antenna locations are then estimated with a maximum error of $1 \mathrm{dm}$.

During the measurement campaign, RSS-values at seven frequencies were recorded sequentially, at an update rate of $3 \mathrm{~Hz}$. An overview of the radio signal parameters are provided in Table I, where the frequencies, bandwidths, transmit powers, signal types and transmitter locations are available. At least 
four of the signals (three FM-radio and one TV-signal) are located at Linköpings water tower, which is around $3.5 \mathrm{~km}$ away at bearing of approximately $80^{\circ}$ from the measurement sites. Two radio-signals are transmitted from the University Hospital in Linköping (US) which is located at a distance of around $3 \mathrm{~km}$ and the bearing to these differs approximately $5^{\circ}$ from the transmitters at the water tower. One transmitter is located almost directly north at a distance of over $20 \mathrm{~km}$.

Initially it was planned to use a Segway RMP 50 robot carrying a SICK laser for navigation to collect the RSS data using laser-SLAM for positioning of the antennas. Unfortunately, it was discovered that the SICK broadcast wide-band noise in the radio spectrum. In verifying measurements up to $10 \mathrm{dBm}$ noise was measured at $1 \mathrm{~m}$ from the SICK effectively burying the signal in noise. Thus, the alternative solution to use the total station was necessary. The experience is reported here to highlight that the navigation method may not be useful for some robotic platforms and that the radio environment of the platform used in the navigation must be duly considered.

\section{B. Antenna}

In these measurements WiFi-antennas were used. The antennas are approximately $3 \mathrm{dm}$ long, and they are likely sleevedipole type antennas. These antennas are far from optimal at FM-radio frequencies where the wavelength is approximately $3 \mathrm{~m}$; hence, the radio signals wavelength is significantly larger than the resonant wavelength of the antenna. Test measurements were performed at a number of FM-frequencies with these antennas, and with a reference antenna (Rohde \& Schwarz HE101), and these indicate an effective antenna gain in the range of -10 to $-20 \mathrm{dBi}$. Clearly, the antenna performs poorly at these frequencies. However, since the FM radio frequencies are likely to be interference-limited rather than noise-limited, and the FM-radio signals are designed so that they can be received also with poor antennas in indoor environments, the signal-to-noise ratio is mostly sufficiently high to enable measurements of RSS values also with this system setup. Furthermore, looking at the targeted applications, the requirement on the antenna size is expected to be even more stringent. Also, larger antennas, such as full-sized dipoles or the reference antenna HE101, would make it more difficult to perform measurements with multiple antennas simultaneously. Considering the size limitations and bandwidth requirement ( $\sim 15 \%$ of the carrier frequency), it is nontrivial to design a passive antenna that is only a small fraction of the wavelength that has a significantly higher effective antenna gain. The interested reader is referred to the classic paper [20] for a discussion on the trade-offs between volume, bandwidth, and antenna efficiency.

\section{Calibration}

At the FM-radio frequencies, the calibration error of four different FunCube Dongle Pro+ radios were evaluated using a Rohde \& Schwarz signal generator (SMBV100A) that generated a sinusoidal signal with a constant output power level of $-60 \mathrm{dBm}$. The resulting calibration error was within

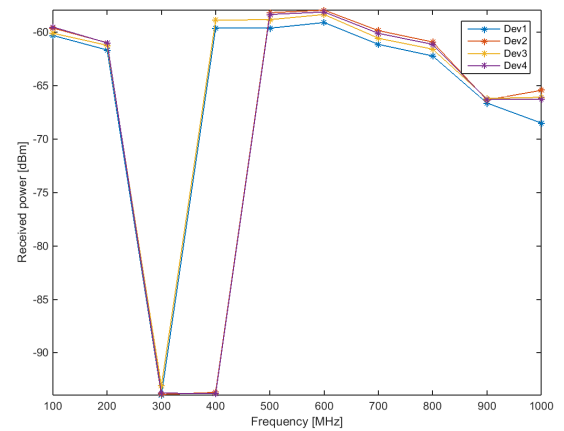

Fig. 1: Received power at calibration frequencies between $100 \mathrm{MHz}$ and $1 \mathrm{GHz}$.

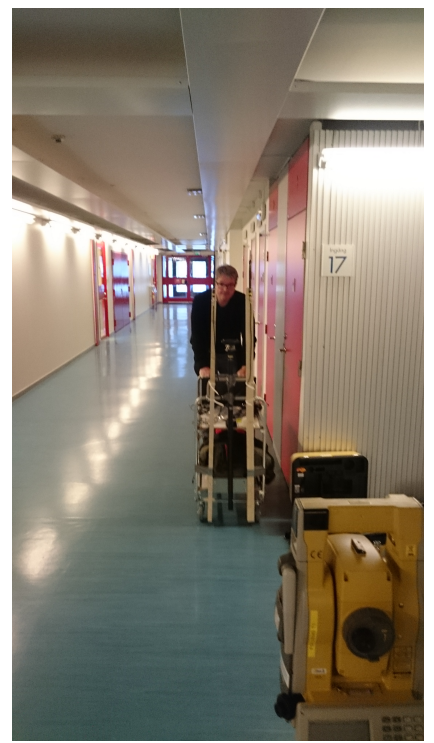

Fig. 2: Illustrations of the equipment used in the experiments.

$\pm 1.5 \mathrm{dBm}$ for all four units over the FM-radio band, and the largest difference between them was slightly over $1 \mathrm{dBm}$ at any individual frequency. Although this calibration was not comprehensive, since it does not examine potentially nonlinear behavior at other signal levels, it still gives an indication of the performance between different receivers. The four receivers were purchased at different times and their serial numbers indicate that they are from different production batches. The design has altered somewhat between them which explains the different behavior of the pass band filters for the two batches at the measured frequency of $400 \mathrm{MHz}$. The received power for the four antennas at the calibration frequencies are shown in Fig. 1. The equipment used in the experiments performed can be seen in Fig. 2.

\section{Collected Data}

The collected data is made publicly available on ResearchGate [21]. The data is provided as raw data since the synchronization between the total station and the collected RSS signals is an open issue. Also a best effort synchronized data 
TABLE I: Measurement frequencies and signal characteristics. (Source: http://www.teracom.se and www.radioguiden.se.)

\begin{tabular}{|c|c|c|c|c|}
\hline $\begin{array}{c}\text { Frequency } \\
{[\mathrm{MHz}]}\end{array}$ & $\begin{array}{c}\text { Bandwidth } \\
{[\mathrm{kHz}]}\end{array}$ & $\begin{array}{c}\text { Transmit power } \\
{[\mathrm{W}]}\end{array}$ & Signal type and station & Transmitter location \\
\hline 94.4 & 120 & 500 & FM radio, Bandit Rock & Water tower $\left(58^{\circ} 23^{\prime} 57^{\prime \prime} \mathrm{N}, 15^{\circ} 38^{\prime} 06^{\prime \prime} \mathrm{E}\right)$ \\
\hline 95.5 & 120 & 490 & FM radio, Linköping community radio & University Hospital $\left(58^{\circ} 24^{\prime} 11^{\prime \prime} \mathrm{N}, 15^{\circ} 37^{\prime} 13^{\prime \prime} \mathrm{E}\right)$ \\
\hline 97.3 & 120 & 150 & FM radio, $\mathrm{SR}$ P3 & Water tower \\
\hline 99.8 & 120 & 500 & FM radio, SR P4 & Water tower \\
\hline 103.2 & 120 & 500 & FM radio, East FM & University Hospital $\left(58^{\circ} 24^{\prime} 06^{\prime \prime} \mathrm{N}, 15^{\circ} 37^{\prime} 15^{\prime \prime} \mathrm{E}\right)$ \\
\hline 106.9 & 120 & 7000 & FM radio, Mix Megapol & Högsäter-Rörstorp $\left(58^{\circ} 33^{\prime} 56^{\prime \prime} \mathrm{N}, 15^{\circ} 31^{\prime} 48^{\prime \prime} \mathrm{E}\right)$ \\
\hline 474.0 & 7000 & Not available & TV, channel 21 (net 3 ) & Water tower \\
\hline
\end{tabular}

will be made available for direct use. The synchronized data is constructed from positions from the total station interpolated to match the time of the collected RSS data. The synchronization is based on captured keyboard input signifying the start of motion from endpoints of the corridors measured. The data has been collected at four sites one of which is a small collection of outdoor data. Two of the sites consist of several corridors of similar university buildings while more extensive measurements have been collected in an evacuated office corridor undergoing refurbishment. The university measurements were made during off peak examination period so less students than normal were present. Still, some students were moving around in the corridors during the measurements probably affecting the data. One of the university sites have measurements in corridors that overlap with the experiments in [14] providing the possibility to analyze these results further.

An illustration of where the data has been collected can be found in Fig. 3, where typical RSS measurements are used to indicate where measurements were taken.

\section{DATA AnAlysis}

One motivation for this paper was the good SLAM results of the thesis work in [22], also reported in [14], and the need to better understand the underlying signals. Below, the usefulness of GP for the signals and the uniqueness of fingerprints are analysed, leading up an assesment of their use in SLAM especially collaborative SLAM.

\section{A. RSS Analysis}

In Fig. 4, the measured RSS are shown as a function of the $x$-position (along the corridor) for measurment site 3 . In this test, measurements were performed several times back and forth in the middle of the corridor. From these results a few conclusions can be drawn. First of all, considering the validity of the measurements, the RSS at $474 \mathrm{MHz}$ (black lines) at antenna 1 are very low and they do not exhibit the same fading pattern as for antenna 2. This reflection holds for most of the other measurement data sets. Hence, the data for this frequency and antenna is discarded in our further analyses. For all frequencies of three data sets collected at this location the RSS varies from -47 to below $-60 \mathrm{dBm}$, and they exhibit fading with fading minima that are suppressed up to $10 \mathrm{dBm}$. Another observation is that the RSS are quite stable over time during the measurement, where different passages at approximately the same position differs by typically 1 or
$2 \mathrm{dBm}$. These differences also include the differences in $y$ position over the measurements (which are typically within $\pm 1 \mathrm{dm}$ in data set 1 ).

However, although the RSS at the two antennas exhibits fairly similar fading characteristics (spatial correlation) considering the distance between fading minima, the absolute RSS exhibit a larger difference (up to 3 to $4 \mathrm{dBm}$ depending on frequency). This can, for instance, be observed at $106.9 \mathrm{MHz}$ (yellow line) where the RSS are significantly lower at antenna 1. A possible explanation could be antenna coupling which alters the effective antenna diagrams for the two antennas. The antennas are spaced approximately $2 \mathrm{dm}$ from each other and antenna coupling effects between antennas and possibly to the roller-board should be expected. These differences are likely not caused by the difference in $y$-position between the two antennas since this effect should then also be seen when examining the separate walks for a specific antenna. The radio wave propagation is complex and it is difficult to determine the actual causes of the RSS fluctuations, they are most likely the effect of a mix of multi-path (small-scale) and shadow (large-scale) fading. The latter phenomenon is probably to some extent caused by wave propagation effects when passing doorways to office rooms, where most of the doors were open. Hence, it is not feasible to attempt to statistically analyze the small-scale fading effects separately since they are not possible to separate in the measurements. Furthermore, the dynamic range of the receivers have not yet been validated, and the flat peaks of the fading maxima for frequency $106.9 \mathrm{MHz}$ could possibly be caused by receiver saturation. First of all, examining the FM radio frequencies that are being transmitted from the same location (94.4, 97.3 and $99.8 \mathrm{MHz}$ ), their fading patterns are similar as seen in Fig. 4. The RSS at $94.4 \mathrm{MHz}$ are lower than expected considering that the transmit power is claimed to be at the same level as at $99.8 \mathrm{MHz}$. Although the signals are transmitted from the same location, they have a small difference in frequency and their RSS differ somewhat spatially and they can be utilized in the positioning system. Similarly, it is difficult to identify any significant difference in the fading pattern at $106.9 \mathrm{MHz}$, even though this signal arrives from a different direction, see Table I. Now, turning our attention to the $474.0 \mathrm{MHz}$ signal, it experiences a slightly more rapid fading (spatially) than the FM radio signals. However, considering that the wavelength at this frequency is less than a quarter of the wavelength at the FM radio frequencies, the fading distance is surprisingly long. This indicates that the multipath-induced (small-scale) fading 


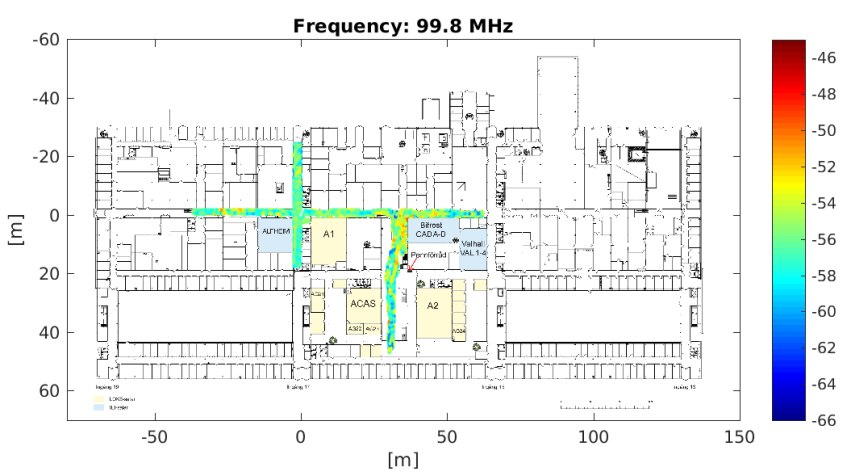

(a) Site 1: A-building, Campus Valla, Linköping University, Linköping, Sweden
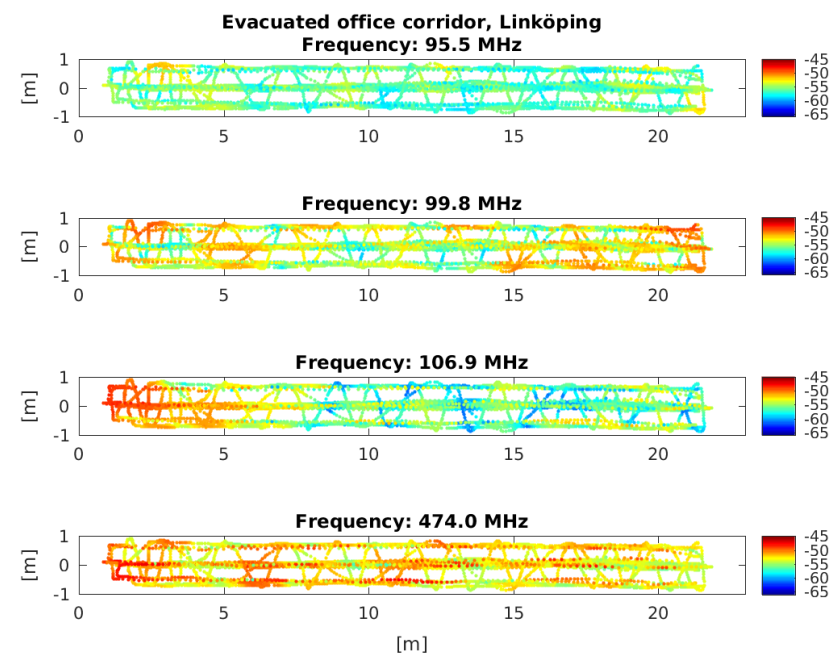

(c) Site 3: Evacuated office corridor, Linköping

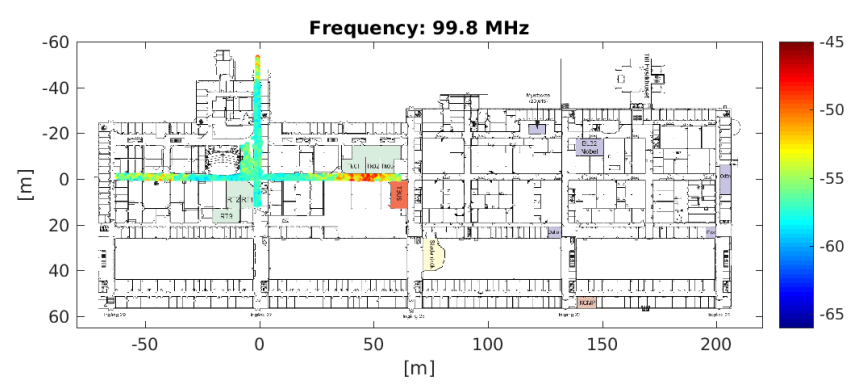

(b) Site 2: B-building, Campus Valla, Linköping University, Linköping, Sweden

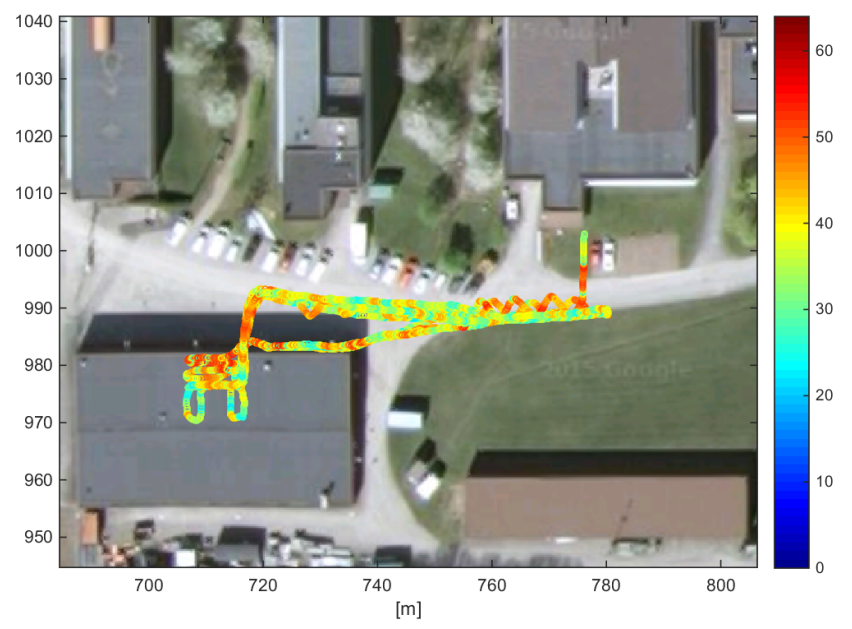

(d) Site 4: Outdoor backyard, Linköping (Google maps (c)2016 Lantmäteriverket/Metria, DigitalGlobe)

Fig. 3: Illustration of the conducted RSS measurements and measurement sites.

may not be the dominating effect. When comparing the RSS levels at the two other buildings, the signal also experiences similar fading characteristics in many parts of the building. However, large-scale patterns in the order of tens of meters around certain areas of the buildings can also be observed.

\section{B. Gaussian Process Validation}

GPs have been generated for one of the corridors in the B-building, Campus Valla, Linköping University, Linköping, Sweden. The GP uses a constant mean function $\mathcal{M}=c$ and the covariance function is assumed to be an isometric squared exponential,

$$
\mathcal{K}\left(x, x^{\prime}\right)=\sigma^{2} e^{-\frac{\left(x-x^{\prime}\right)^{T}\left(x-x^{\prime}\right)}{2 \ell^{2}}},
$$

where $\sigma^{2}$ is the signal variance and $\ell^{2}$ the characteristic signal length. The measurement noise is assumed additive Gaussian with variance $\sigma_{e}^{2}$. In this setup, $c, \sigma, \ell$, and $\sigma_{e}$ are the hyperparameters of the GP.

Three different sets of hyper-parameters have been evaluated. The first set of hyper-parameters have been obtained as the result of minimizing the negative log marginal likelihood with respect to the hyper-parameters. The two remaining sets have been determined by considering a simplified wave propagation model and inspecting the collected raw data. They differ only in the characteristic signal length, $\ell$. The hyperparameters used are presented in Table II.

By training a GP on the majority of measurements consisting of jigg saw motion through one corridor and measurements along the sides and testing its predictivity against measurements in the middle it is evident that the predictions by the GP holds. Thus, in an indoors environment the fast fading 

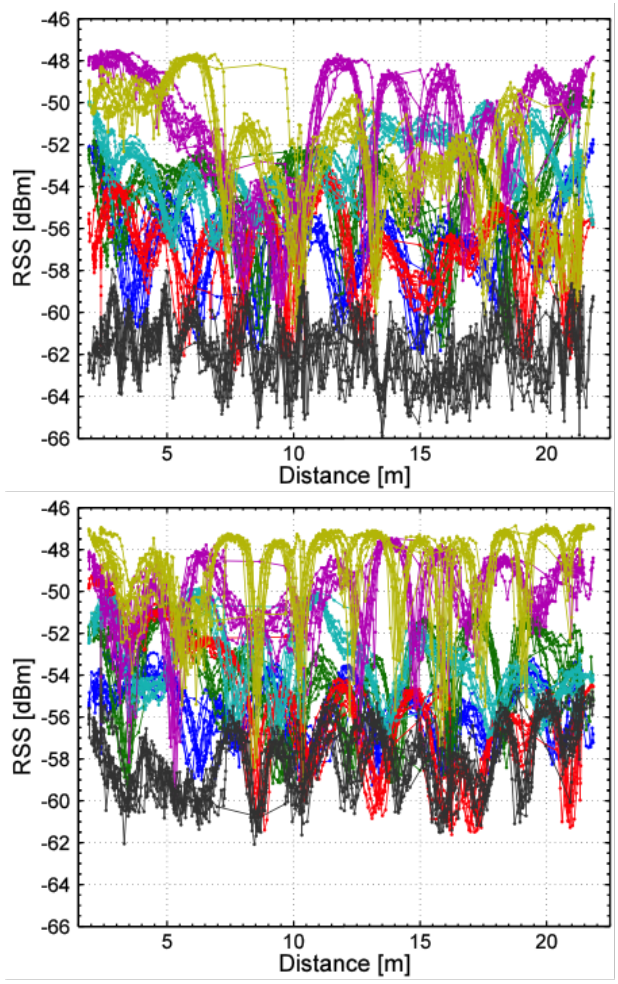

Fig. 4: Measured RSS for data set 1 (at site 3 ) as a function of $x$-position for all seven frequencies. Top: antenna 1. Bottom: antenna 2. Frequencies (in $\mathrm{MHz}$ ): blue -94.4 , green -95.5 , red -97.3 , cyan -99.8 , magenta -103.2 , yellow -106.9 , and black - 474.0 .

TABLE II: Hyper-parameters used to evaluate the suitability of the GP to model the RSS variations.

\begin{tabular}{ccccc}
\hline \multirow{2}{*}{ Set } & \multicolumn{4}{c}{ Hyper-parameters } \\
& $c$ & $\sigma$ & $\ell$ & $\sigma_{e}$ \\
\hline 1 & -54.5258 & 2.2973 & 0.4046 & 0.2898 \\
2 & -55.0000 & 2.5000 & 1.5000 & 0.5000 \\
3 & -55.0000 & 2.5000 & 3.0000 & 0.5000 \\
\hline
\end{tabular}

patterns are relatively stable over time (hours at least) and the GP models fits it well.

In Fig. 5-9 the progression of the GP model toward covering the width of a whole corridor is clear. In Fig. 9(b) the GP exhibits only one single flat mode cross the corridor. The crossvalidation in Fig. 10 with measurements from a straight passage through jigg saw motion measurements look convincing considering that the shaded area represents $2 \sigma$ and a few outliers can be expected. The cross validation with the second antenna in Fig. 11 exhibits a peak where it should have been a dip at approximately $40 \mathrm{~m}$ illustrating that the training data is to sparse to capture all quick cross corridor variations. The fit improves as we progresses to the manual parameters in Fig. 12 and 13 but is always poor around $40 \mathrm{~m}$. The cost of the better results cross the corridor is a more smooth longitudinal variation giving slightly poorer fits in Fig. 14 and Fig. 15.
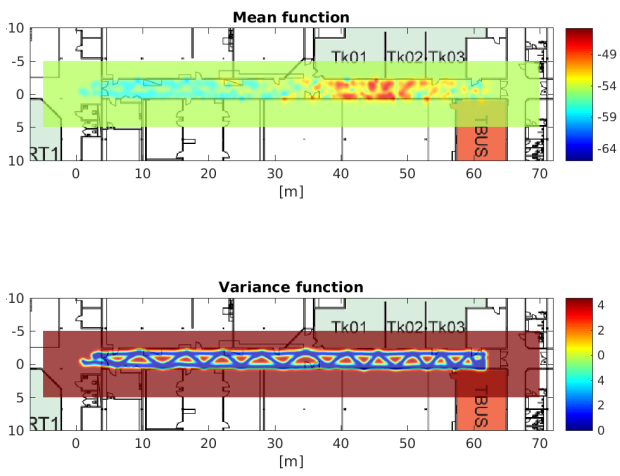

Fig. 5: GP generated for part of the B-building (at site 2), Campus Valla, Linköping University, Linköping, Sweden, data as an illustration of the generated RSS frequency maps using the optimized parameter setting.
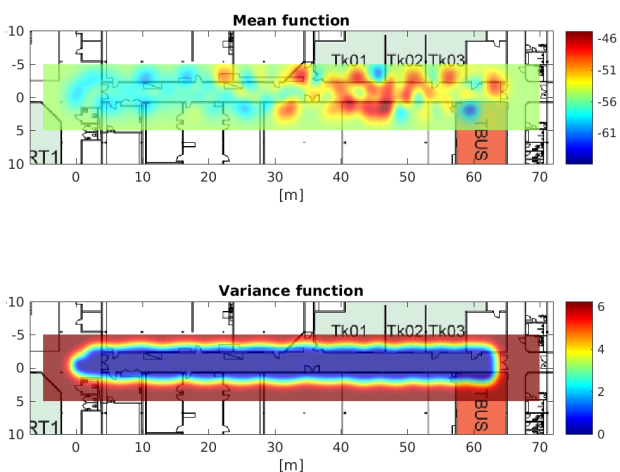

Fig. 6: GP generated for part of the B-building (at site 2), Campus Valla, Linköping University, Linköping, Sweden data as an illustration of the generated RSS frequency maps using parameter set $2(\ell=1.5 \mathrm{~m})$.
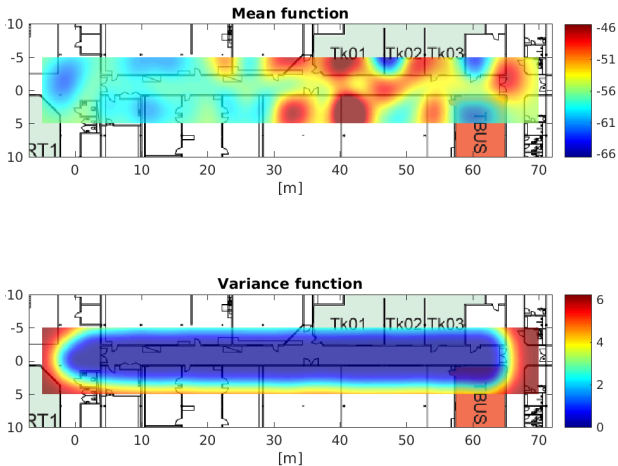

Fig. 7: GP generated for part of the B-building (at site 2), Campus Valla, Linköping University, Linköping, Sweden, data as an illustration of the generated RSS frequency maps using parameter set $3(\ell=3.0 \mathrm{~m})$. 


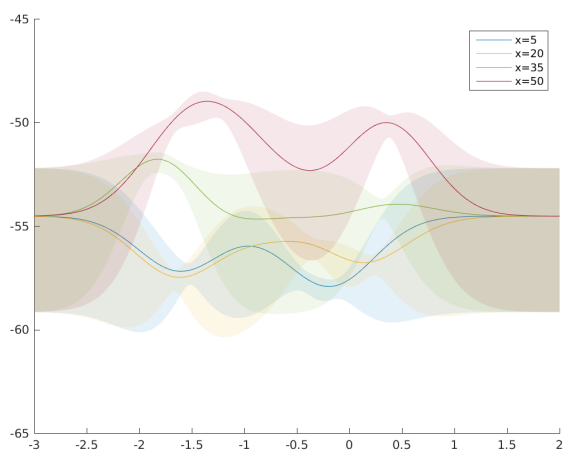

(a) Lateral cross-sections.

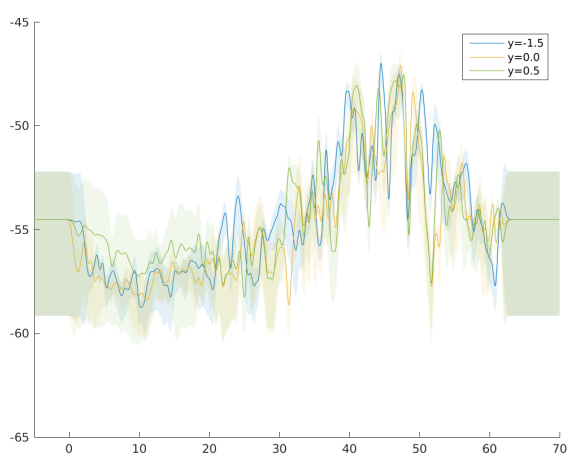

(b) Longitudinal cross-sections.

Fig. 8: Cross-sections of the GP show in Fig. 5. The shaded area represents two standard deviations.

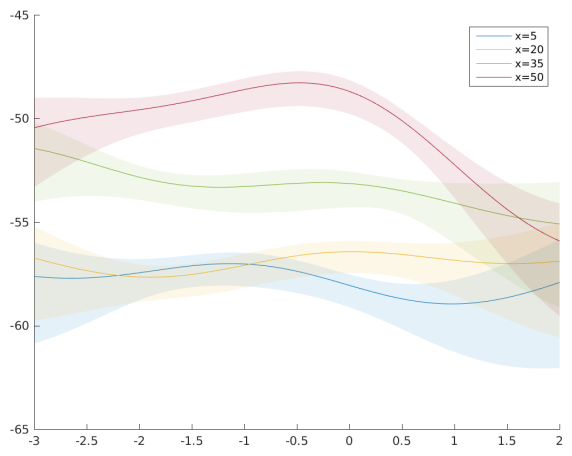

(a) Lateral cross-sections matching Fig. 6.

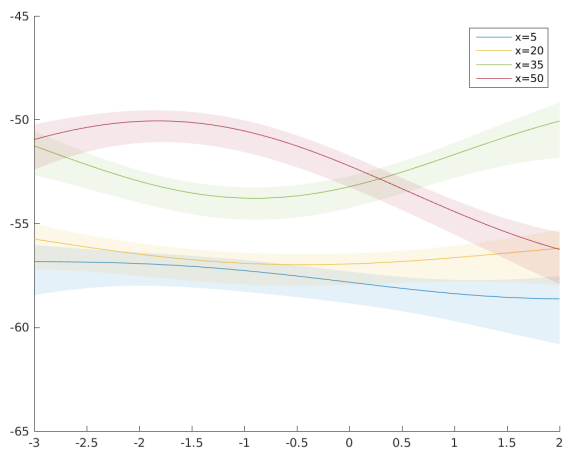

(b) Lateral cross-sections matching Fig. 7.

Fig. 9: Cross-sections of the GPs shown in Fig. 6 and Fig. 7. The shaded area represents two standard deviations

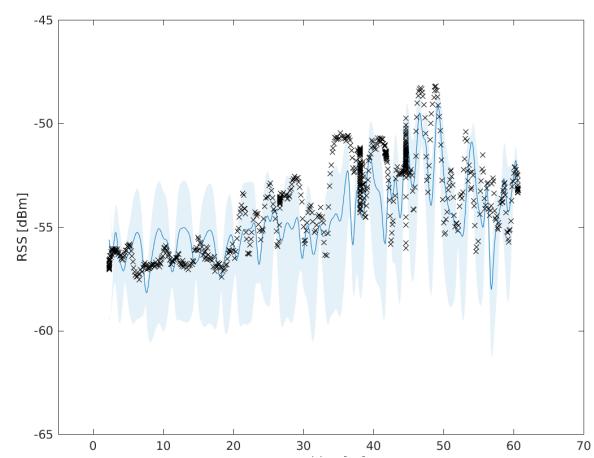

Fig. 10: Crossvalidation of the generated GP shown in Fig. 5. The shaded area represents two standard deviations, and the crosses measurements from the validation data set.

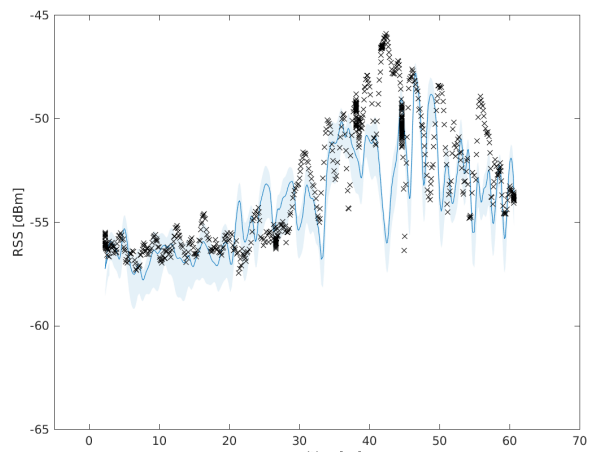

Fig. 11: Crossvalidation based a GP (optimized parameters) generated by all data in Fig. 5, and then validated with a matching subset in the other antenna. The shaded area represents two standard deviations, and the crosses measurements from the validation data set.

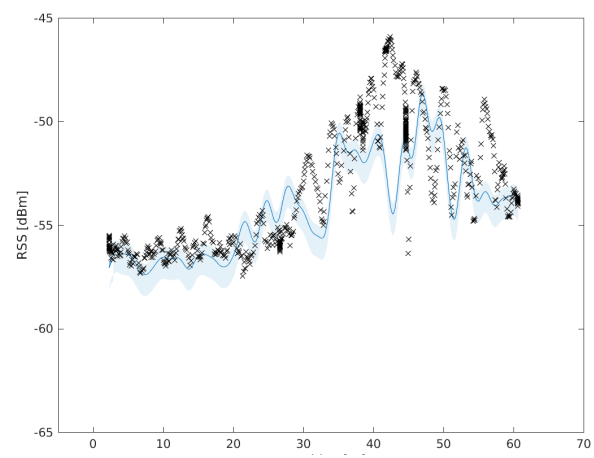

Fig. 12: Crossvalidation based a GP (parameter set 2) generated by all data in Fig. 6, and then validated with a matching subset in the other antenna. The shaded area represents two standard deviations, and the crosses measurements from the validation data set. 


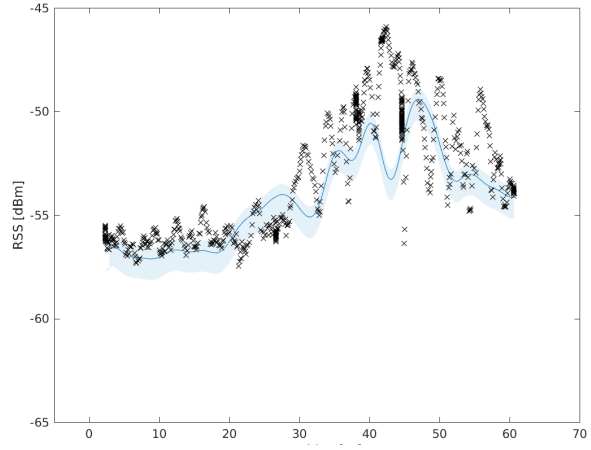

Fig. 13: Crossvalidation based a GP (parameter set 3) generated by all data in Fig. 7, and then validated with a matching subset in the other antenna. The shaded area represents two standard deviations, and the crosses measurements from the validation data set.

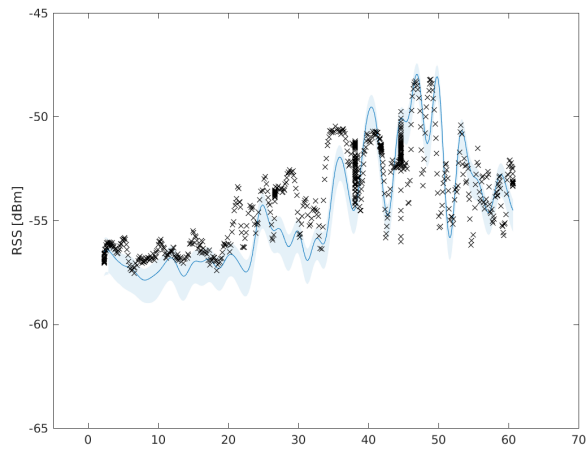

Fig. 14: Crossvalidation of the generated GP shown in Fig. 6. The shaded area represents two standard deviations, and the crosses measurements from the validation data set.

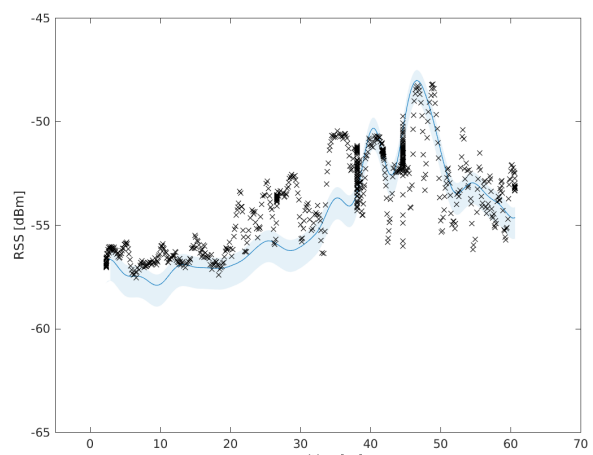

Fig. 15: Crossvalidation of the generated GP shown in Fig. 7. The shaded area represents two standard deviations, and the crosses measurements from the validation data set.
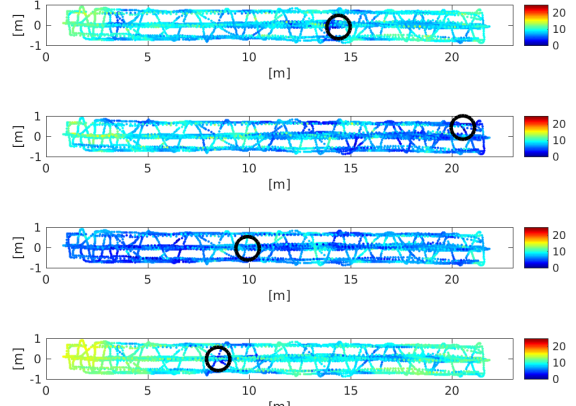

Fig. 16: Finger print norm for FOI corridor.
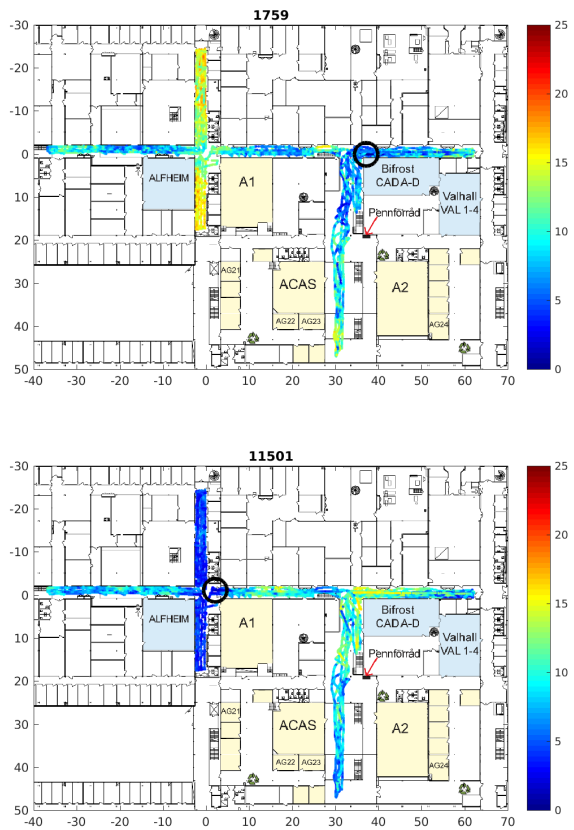

Fig. 17: Finger print norm for corridor in the A-building (at site 1), Campus Valla, Linköping University, Linköping, Sweden.

\section{Uniqueness of Fingerprints}

The uniqueness of the 7 frequency fingerprints were tested by calculating the norm to all other measurements from random locations, see Fig. 16-18. A few sites have a unique fingerprint but in the majority of cases the fingerprint can be reasonably well matched in more places than one resulting in multimodal posterior densities. This indicate that batch solutions that are working with the whole sequence, or other methods working on the whole succession of fingerprints, such as the particle filter solution in [14], and can handle temporary position ambiguities are probably more successful. As noted in [23] deep fades from fast fading effects could present a challenge for single fingerprints. However, if the variations are stable and can be modeled witihn the SLAM solution they instead can be used to increase the resolution. 

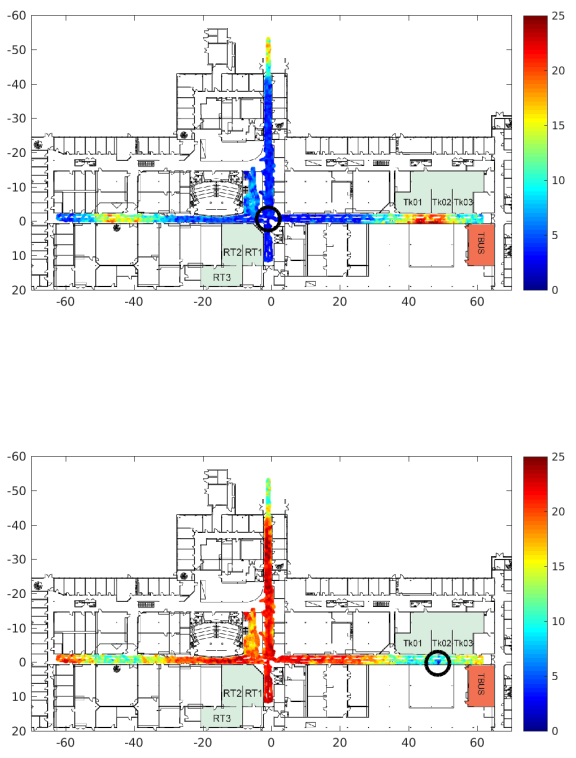

Fig. 18: Finger print norm for corridor in the B-building (at site 2), Campus Valla, Linköping University, Linköping, Sweden.
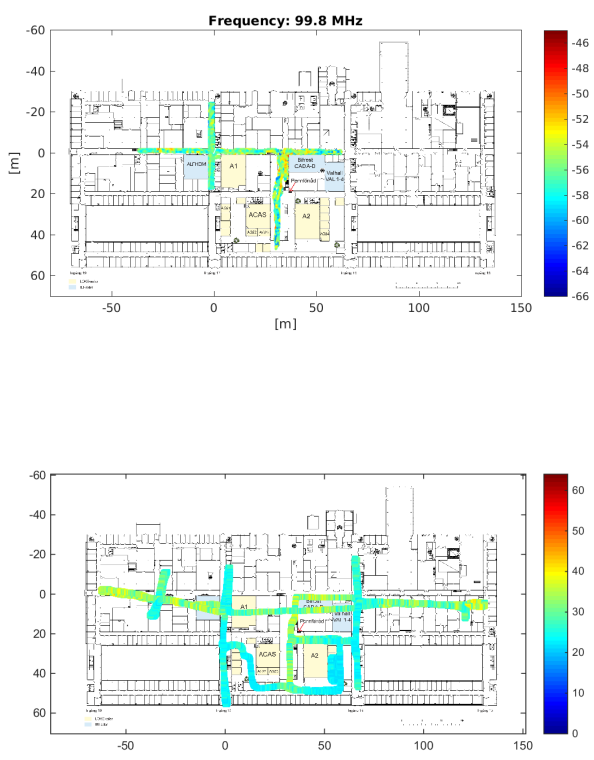

Fig. 19: New measurment trails compared with RSS-SLAM solution from earlier IPIN 2014 results

\section{Consequences for SLAM}

For the optimized hyper-parameters GP the optimum characteristic signal length is short, much shorter than the one used in [14]. The lateral variation in an ordinary corridor as in Fig. 3(c) could pose trouble for collaborative navigation if the teammates do not overlap tracks often enough since the information is rather localized. It is possible that using the larger scale fading pattern of length $3-4 \mathrm{~m}$, as predicted by the FM-signal wavelength, allowed generalization of the collected measurements so that the lateral variations were discounted and mainly the larger scale longitudinal variations along the corridors affected the solution in [14]. A rather unexpected behavior by the filter in [14] was that on a straight path the SLAM would correct position cross the path even though the map would essentially only capture variations along the path. However, if we consider the variance of the GP it will in essence put a premium on paths continuing in the traveled direction as long as the measurements fit the predictions. Thus, it introduces an implicit bias for corridor following by putting a prior on such motion.

The fading characteristics are a mix of small- and largescale fading effects. As shown in [14], where a foot-mounted INS was integrated with multi-frequency RSS measurements, the SLAM algorithm was able to efficiently make use of the RSS data. The fading minima are relatively wide in the spatial domain in these measurements, on the metre-level, as seen in Fig. 18. Hence, the fading minima can be efficiently exploited by the SLAM algorithm, and makes up a valuable contribution when used in a multi-frequency measurement setting. This is in contrast to what has been reported at higher frequencies, such as Bluetooth Low Energy (BLE) at $2.4 \mathrm{GHz}$ [23], where the spatially focused fading minima (on a decimetre-level) are more difficult to exploit. A visual inspection of data from the previous measurements in Fig. 19 indicates that the large scale variations are robust over longer periods. Remark, only the variations can be validated since the measurments in [14] was collected with a different gain so the scale and level have to be adjusted.

\section{CONCLUSIONS}

This paper presents a data collection effort investigating the usefulness of multi-frequency RSS for indoor localization.

A collected set of data from four sites is presented. There is a variation in RSS on the length of $0.4 \mathrm{~m}$. The variations are repeatable over the recorded time spans (order of hours).

For collaborative navigation it is doubtful if the resolution made possible by these variations can be leveraged since the cross path variations are not well covered. The small variations can also be hidden by other variations due to shadowing by the body of the antenna, among other.

The use of GPs to model the variations have been analyzed showing a good potential in capturing the variations of the RSS signals. To hand tune the hyper-parameters may be better than to optimize them depending on the scenario, i.e., if the path variation is low in a team and the second team member can 
traverse the other side of the corridor a more robust model is needed.

The fingerprints of the chosen frequencies are not necessarily unique but show good prospects for use in smoothing or smoothing-like algorithms where the sequence of measurements come into play.

\section{ACKNOWLEDGEMENT}

This work was funded by the Swedish Armed Forces through the Command and Control R\&D program, reference AF.9220209. The work has also been supported by the project Cooperative Localization (CoopLoc) funded by Swedish Foundation for Strategic Research (SSF), the Swedish strategic research center Security Link and the Vinnova Industry Excellence Center LINK-SIC

\section{REFERENCES}

[1] J. Rantakokko, J. Rydell, P. Stromback, P. Handel, J. Callmer, D. Tornqvist, F. Gustafsson, M. Jobs, and M. Gruden, "Accurate and reliable soldier and first responder indoor positioning: multisensor systems and cooperative localization," IEEE Transactions on Wireless Communications, vol. 18, no. 2, pp. 10-18, Apr. 2011.

[2] J.-O. Nilsson, J. Rantakokko, P. Händel, I. Skog, M. Ohlsson, and K. Hari, "Accurate indoor positioning of firefighters using dual footmounted inertial sensors and inter-agent ranging," in Proceedings of Position Location and Navigation Symposium (PLANS), April 2014.

[3] B. Ferris, D. Fox, and N. Lawrence, "WiFi-SLAM using Gaussian process latent variable models," in Proceedings of the 20th International Joint Conference on Artifical Intelligence (IJCAI), Hyderabad, India, Jan. 2007.

[4] J. Huang, D. Millman, M. Quigley, D. Stavens, S. Thrun, and A. Aggrwal, "Effient, generalized indoor WiFi GraphSLAM," in 2011 IEEE International Conference on Robotics and Automation (ICRA), Shanghai, May 2011.

[5] P. Addesso, L. Bruno, and R. Restaino, "Integrated RSS from unknown access points in wlan positioning," in 2011 7th International Wireless Communications and Mobile Computing Conference (IWCMC), Istabul, Turkey, Jul. 2011, pp. 607-612.

[6] V. Moghtadaiee, A. Dempster, and S. Lim, "Indoor localization using FM radio signals: A fingerprinting approach," in 2011 International Conference on Indoor Positioning and Indoor Navigation (IPIN), Sep. 2011, pp. 1-7.

[7] Y. Chen, D. Lymberopoulos, J. Liu, and B. Priyantha, "FM-based indoor localization," in Proceedings of the 10th international conference on Mobile systems, applications, and services. ACM, 2012, pp. 169-182.

[8] A. Popleteev, V. Osmani, and O. Mayora, "Investigation of indoor localization with ambient fm radio stations," in Proceedings of PerCom, Lugano, Switzerland, March 2012.

[9] R. Faragher, C. Sarno, and M. Newman, "Opportunistic radio SLAM for indoor navigation using smartphone sensors," in 2012 IEEE/ION Position Location and Navigation Symposium (PLANS), Apr. 2012, pp. 120-128.

[10] P. Bahl and V. N. Padmanabhan, "RADAR: An in-building RF-based user location and tracking system," in Proceedings of 19th Annual Joint Conference of the IEEE Computer and Communications Societies (INFOCOM), Tel Aviv, Israel, Mar. 2000.

[11] H. Hashemi, "The indoor radio propagation channel," Proceedings of the IEEE, vol. 81, no. 7, pp. 943-968, Jul 1993.

[12] S. Rice, "Mathematical analysis of random noise," The Bell System Technical Journal, vol. 23, no. 3, pp. 282-332, Jul 1944.

[13] B. Ferris, D. Hähnel, and D. Fox, "Gaussian processes for signal strength-based location estimation," in In Proc. of Robotics Science and Systems, 2006.

[14] M. Nilsson, J. Rantakokko, M. A. Skoglund, and G. Hendeby, "Indoor positioning using multi-frequency RSS with foot-mounted INS," in Fifth International Conference on Indoor Positioning and Indoor Navigation, 2014.

[15] J. Fink and V. Kumar, "Online methods for radio signal mapping with mobile robots," in 2011 IEEE International Conference on Robotics and Automation (ICRA), Shanghai, May 2011.
[16] P. Eliardsson, U. Uppman, and J. Rantakokko, "Received signal strength measurements utilizing signals of opportunity - a feasibility study on the use of RSS for loop-closure in multisensor indoor positioning systems,” FOI, Tech. Rep. FOI-R-3841-SE, Feb. 2014.

[17] C. E. Rasmussen and C. K. I. Williams, Gaussian processes for Machine Learning. MIT Press, 2006.

[18] A. Ranganathan, M.-H. Yang, and J. Ho, "Online sparse gaussian process regression and its applications," IEEE Transactions on Image Processing, vol. 20, no. 2, pp. 391-404, 2011.

[19] J. Quinonero-Candela, C. E. Rasmussen, and C. K. Williams, "Approximation methods for Gaussian process regression," Large-scale kernel machines, pp. 203-223, 2007.

[20] H. Wheeler, "Small antennas," Antennas and Propagation, IEEE Transactions on, vol. 23, no. 4, pp. 462-469, Jul 1975.

[21] "ResearchGate data-Repository," https://www.researchgate.net/ publication/299989538_Indoor_Localization_Using_Multi-Frequency_ RSS, accessed: Apr., 08, 2016.

[22] M. Nilsson, "Indoor positioning using opportunistic multi-frequency RSS with foot-mounted INS," Master's thesis, Linköping University, 2014.

[23] R. Faragher and R. Harle, "An analysis of the accuracy of bluetooth low energy for indoor positioning applications," in Proceedings of the 27th International Technical Meeting of the Satellite Division of the Institute of Navigation (ION GNSS+'14), 2014. 